\title{
Status of the experiment for the search of a dark photon at VEPP-3
}

\author{
I. Rachek ${ }^{1, a}$, D. Nikolenko ${ }^{1}$, and B. Wojtsekhowski ${ }^{2}$ \\ ${ }^{1}$ Budker Institute of Nuclear Physics, 630090 Novosibirsk, Russia \\ 2 Jefferson Laboratory, Newport News, VA, USA
}

\begin{abstract}
The status of the experiment to search for a new gauge boson, $\mathrm{A}^{\prime}$, in $e^{+} e^{-}$annihilation by means of a positron beam incident on a gas hydrogen target internal to the bypass at the VEPP-3 storage ring is reported. The search method is based on a missing mass spectra in reaction $e^{+} e^{-} \rightarrow \gamma \mathrm{A}^{\prime}$. It allows observation of the $\mathrm{A}^{\prime}$ signal independently of its decay modes and life time. The projected result of this experiment corresponds to an upper limit on the square of coupling constant $\varepsilon^{2}=(0.2 \div 1.0) \cdot 10^{-7}$ with a signal-to-noise ratio of two to one at $\mathrm{A}^{\prime}$ mass of $5-20 \mathrm{MeV}$.
\end{abstract}

\section{Introduction}

The search for an experimental signature of physics beyond the Standard Model is a major effort of modern particle physics. The existence of Dark Matter (DM) is supported by many astrophysical data. At first, weaklyinteracting massive particles (WIMPs) were considered as main candidates for DM. However, up to now no evidence for WIMPs at LHC or in direct detection experiments has been reported. That has initiated a hunt in a wider area and sparked a renewed interest in searching for a new gauge boson, the so-called dark photon or $\mathrm{A}^{\prime}$-boson, which mediates interactions between DM particles, as well as between DM particles and Standard Model particles through the kinetic mixing mechanism [1]. In recent years, considerable efforts have been devoted to the re-analysis of existing data in attempts to observe a manifestation of a new boson. An extended review of the present status of such searches can be found in the recent report [2]. So far, no evidence of new particles has been found. In parallel, a series of new dedicated experiments has been proposed.

Here we present the status of the experiment to search for a dark photon, proposed to be conducted at the VEPP-3 electron/positron storage ring at the Budker Institute, Novosibirsk.

\section{The concept}

The concept of the method is partly described in [3]. A positron beam in a storage ring with an energy $E_{+}$of a few hundred $\mathrm{MeV}$ and an internal hydrogen gas target make up an " $e^{+} e^{-}$-collider". In such a collider it is possible to search for the production of a light $\mathrm{A}^{\prime}$-boson with a mass of up to $m_{\mathrm{A}^{\prime}}[\mathrm{MeV}] \sim \sqrt{E_{+}[\mathrm{MeV}]}$. Unlike many other experiments with a fixed target, which are based on the detection of $e^{-} e^{+}$or $\mu^{+} \mu^{-}$pairs from $\mathrm{A}^{\prime}$-boson decay, in the

\footnotetext{
a e-mail: rachek@inp.nsk.su
}

described experiment no special assumptions about decay modes of the $\mathrm{A}^{\prime}$-boson are required.

Specifically for the VEPP-3 storage ring an experimental approach looks as follows:

- A positron beam in VEPP-3 repeatedly crosses an internal gas target. The target is a cooled windowless storage cell filled with hydrogen gas.

- VEPP-3 will work with the new injection complex, commissioned recently at the Budker Institute. The new injector is capable of providing $2 \times 10^{9}$ positrons per second. Besides linear accelerators, it contains a damping ring which can be used as a positron booster storage ring for VEPP-3. The switching between old and new injection facilities at VEPP-3 is ongoing.

- The beam energy will be equal to the injector energy, which is up to $500 \mathrm{MeV}$, so no energy ramping in VEPP-3 is envisaged. This ensures the highest luminosity.

- A six-bunch regime of VEPP-3 operation will be implemented. Bunches are equally spaced in the VEPP-3 orbit, and every 10 seconds the oldest bunch is replaced by a new one, which was accumulated in the injector dumping ring. Thus, a luminosity of up to $3 \times 10^{33} \mathrm{~cm}^{-2} \mathrm{~s}^{-1}$ can be achieved.

- A special magnet will be installed downstream from the target to turn away the positron beam and thus to allow a free flight for gamma-quanta from target to photon detector.

- A segmented EM-calorimeter will be used to detect photons.

- To control the systematics a run with the electron beam in VEPP-3 can be performed, thus making sure that a putative boson is seen only with a positron beam. 


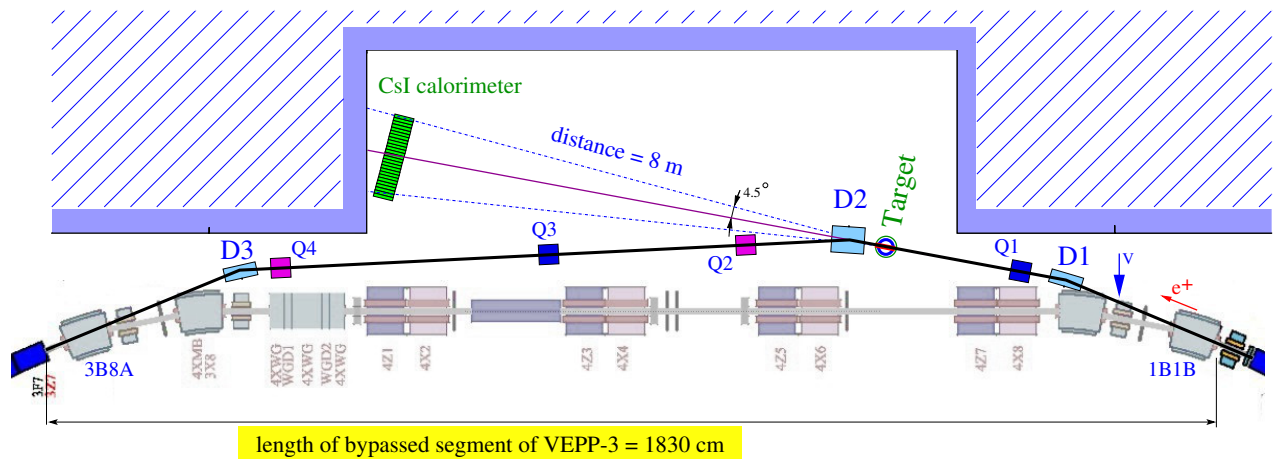

Figure 1. The layout of the proposed experiment with a bypass built along the straight section of the VEPP-3 ring. $D 1-D 3-$ dipole magnets, $Q 1-Q 4$ - quadrupole lenses.

\section{The proposed experimental setup}

VEPP-3 is a buster-ring, operating as an intermediate accelerator/storage ring of electrons and positrons for the VEPP-4 collider. There are time slots when VEPP-3 is available for other experimental programs, including the electro/photo-nuclear experiments, utilizing a super-thin internal target technique.

Usually the internal target is located in the first 12meter-long straight section of the VEPP-3 ring. In the same straight section there are also two RF cavities, four quadrupole and one sextupole lens and elements of beam injection and extraction. The space available for the internal target equipment is $217 \mathrm{~cm}$ long. In our earlier proposal [4] we have described a possible configuration of the experiment based on a chicane magnet installed in this segment of VEPP-3. However, such a configuration has significant difficulties, both technical and organizational. The latter follows from the fact that when a chicane magnet is installed in the ring, VEPP-3 becomes completely inaccessible to other working regimes, including operation as a booster for VEPP-4, or as a synchrotron radiation source.

Therefore, for the proposed experiment it was decided to implement a special setup. The main element of this setup is a bypass, which has to be built along the second straight section of the VEPP-3 ring.

\subsection{Bypass}

The proposed configuration of the bypass is shown in Figure 1 . It consists of $i$ ) a vacuum chamber with a total length of about $18 \mathrm{~m}$; ii) vacuum pumps; iii) three dipole magnets with a combined bending angle of $45^{\circ}$; $i v$ ) a number of quadrupole lenses; $v$ ) elements of beam diagnostics; $v i$ ) an internal target section containing a thin-walled storage cell and set of powerful vacuum pumps.

The bypass will occupy the place which several decades ago was already used for another bypass, containing the Free Electron Laser. FEL has been dismounted, but some elements providing its operation at VEPP-3 (switching magnets, inlet/outlet vacuum channels) are still there. This should simplify significantly the commissioning of the new bypass.

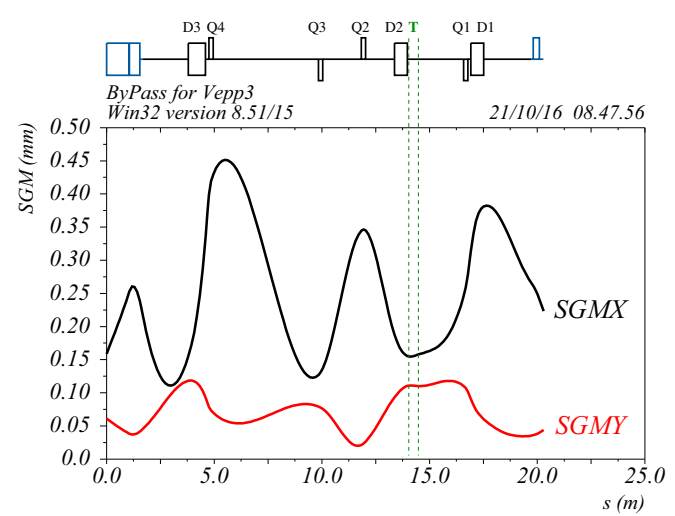

Figure 2. Calculated beam size ( $\mathrm{X}$ and $\mathrm{Y})$ along the bypass for the proposed electron optics. Vertical lines show the location of the internal target.

The R\&D of the bypass electron optics has been recently completed, see Fig. 2, and detailed specifications of the magnetic elements of the bypass have been obtained.

\subsection{Internal target}

A thin-walled open-ended storage cell cooled to $25^{\circ} \mathrm{K}$ and filled with hydrogen gas will be used as an internal target. The magnetic structure of the bypass is designed in such a way as to provide small values of beta-functions in the location of the storage cell, Fig. 2, allowing the use of a small-opening cell. Together with cell cooling this permits us to obtain the required target thickness of about $10^{16} \mathrm{at} / \mathrm{cm}^{2}$ with a smaller amount of hydrogen gas injected into the target. The gas leaks out of the cell ends into the ring vacuum chamber and must be pumped out promptly. A set of powerful turbomolecular and cryogenic pumps will be installed in the target chamber, as well as upstream and downstream from the target chamber. 


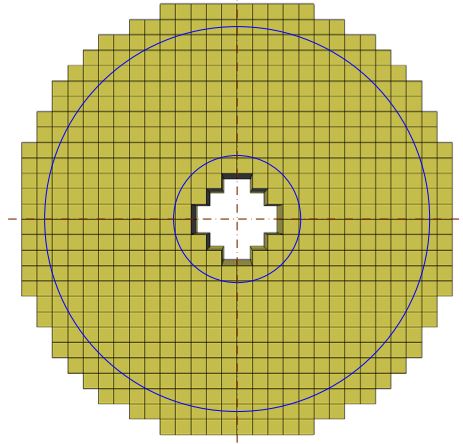

Figure 3. Calorimeter configuration: $624 \mathrm{CsI}(\mathrm{Tl})$ crystals from the CLEO-II endcap calorimeter assembled in a ring shape.

\subsection{Photon detector}

The photon detector can be placed at a distance of up to $8 \mathrm{~m}$ from the target. The requirements for the detector are:

- Energy resolution on the level of $\sigma_{E} / E=5 \%$ for photons with energy $E_{\gamma}=100-450 \mathrm{MeV}$.

- Angular resolution on a level of $0.1^{\circ}$.

- Angular acceptance as defined by the requirement to detect both photons from two-photon annihilation:

- in $\theta$ : symmetrical range in $\theta_{\gamma}^{C M}$ around $90^{\circ}$, e.g. $\theta_{\gamma}^{C M}=60^{\circ}-120^{\circ}$, which corresponds to $\theta_{\gamma}^{L A B}=$ $1.5^{\circ}-4.5^{\circ}$.

- in $\phi:$ total $2 \pi$ coverage.

- The detector should be able to sustain a moderate total photon rate at a level of $1 \mathrm{MHz}$.

A calorimeter with sufficient energy resolution could be constructed using crystals from the discarded CLEO-II detector [5] from Cornell University. It is especially effective because, the Cornell group is considering a similar experiment with a $5 \mathrm{GeV}$ positron beam [6]. The collaboration between the Budker Institute and Cornell University for development of such a detector is already organized [7].

The end-cap electromagnetic calorimeter of the CLEO-II detector consists of $1600 \mathrm{CsI}(\mathrm{Tl})$ crystals of $5 \times 5 \times 30 \mathrm{~cm}^{3}$ size $\left(16.2 X_{0}\right)$. It was used to measure electron and photon energy in a wide range, and a direct measurement of its performance at a photon energy of interest for the proposed experiment, $\mathrm{E}_{\gamma}=180 \mathrm{MeV}$, is available: energy resolution $\delta E / E=3.8 \%$; spacial resolution $\delta x=12 \mathrm{~mm}$, which results in angular resolution of $\delta \theta=1.5 \mathrm{mrad}$ for a distance of $8 \mathrm{~m}$.

In this case it would take 624 crystals to cover the required angular range, see Fig. 3. A few notes on this detector option should be mentioned:

- The CLEO-II calorimeter assembly is clearly inappropriate for the proposed experiment, so a new mechanical support must be designed and constructed. This was recently completed.
- CsI(Tl) crystal has a long light emitting time. Therefore, its ability to work at a high background rate is limited. However, due to the high segmentation of the calorimeter, a long output pulse seems not to be a problem. Even for crystals covering the lowest polar angle, the expected rate of background photons is estimated to be at a level of a few tens $\mathrm{kHz}$ for the projected luminosity of $10^{33} \mathrm{~cm}^{-2} \mathrm{~s}^{-1}$.

The experiment will require a careful account of the detector responses. The energy response will be calibrated by using $\gamma \gamma$ coincidence events produced with the hydrogen target. These data will also provide a detector line shape determination. The use of the electron beam instead of the positron beam provides the way to obtain the "white" photon spectra without the $\mathrm{A}^{\prime}$-boson signal and the two-photon line.

\subsection{Positron veto counter}

The main single-photon QED background comes from positron bremsstrahlung on hydrogen and from the radiative Bhabha process. Therefore, a rejection of such background events with an efficiency $\epsilon$ would result in an increase of the search sensitivity by a factor of $1 / \sqrt{1-\epsilon}$. Since in these processes the positron loses energy and is swept out by the D2 dipole magnet, such background events can be vetoed by detecting the scattered positron. For this purpose, a compact sandwich will be installed downstream from the D2 magnet. The fraction of bremsstrahlung events detected by this counter depends on its geometry. The total rate of positrons emitting a photon with energy above a $E_{\gamma}=50 \mathrm{MeV}$ threshold can be estimated to be $60 \mathrm{MHz}$ for the luminosity of $L=10^{33} \mathrm{~cm}^{-2} \mathrm{~s}^{-1}$. Hence, the veto counter would be useless at this luminosity if it detects all positrons including those which emit photons outside the photon detector acceptance. Using the MC simulation we have chosen a configuration with a positron rate of $f_{e^{+}} \sim 2.5 \mathrm{MHz}$, which gives only $10 \%$ event loss due to accidental coincidences - see Fig. 4. The simulation has shown that the veto efficiency is a function of missing mass range and it is generally higher for the bremsstrahlung from a hydrogen target process than for the radiative Bhabha one, with overall mean value of about $70 \%$.

However, in the case of a visible decay of $\mathrm{A}^{\prime}$ to an electron/positron pair the use of such a veto counter results in a loss of signal events because positrons from the A' decay often hit the veto counter. We have considered a simple extension - a layer of scintillation strips placed in front of the veto sandwich, Fig. 5. This allows us to reconstruct a positron energy and, together with photon energy, reconstructed in the calorimeter, allows us to distinguish between the $\mathrm{A}^{\prime}$ decay process and the bremsstrahlung on the proton. However, the veto efficiency for radiative Bhabha events is rather low in this case. Nevertheless, such a relatively inexpensive configuration of the veto counter does improve the search sensitivity and we are going to implement it. 

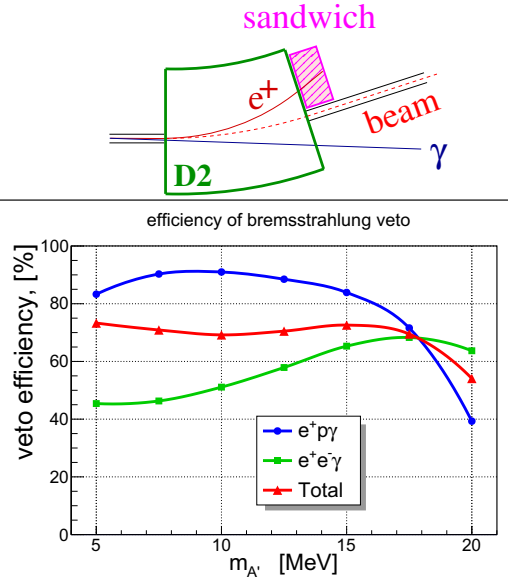

Figure 4. Schematic layout of the positron veto detector placed behind the D2 dipole magnet, and its efficiency as a function of missing mass.

\section{layer of scint. strips}
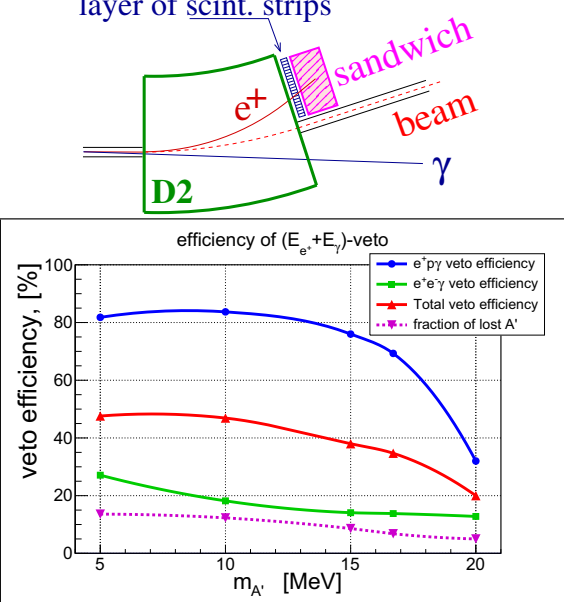

Figure 5. Positron veto detector with a layer of scintillation strips, capable to reconstruct positron energy, and its efficiency as a function of missing mass.

\subsection{Background from QED annihilation process}

Conventional QED $e^{+} e^{-}$annihilation processes have two or more gamma quanta in the final state, while in the signal process there is strictly one photon. This can be used to reject such background.

For this purpose the acceptance of the photon calorimeter is chosen to be symmetrical with respect to $\theta_{\gamma}^{c m}=90^{\circ}$ in the center of mass frame of an electron/positron pair. This means that for the 2-photon annihilation, the calorimeter will always detect either both photons or none. Therefore, the 2-photon annihilation events will be rejected very effectively. Three-photons events will also be largely rejected.

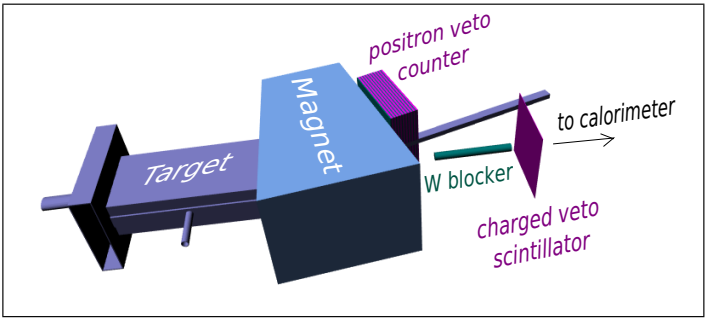

Figure 6. Charged veto scintillator behind the tungsten "blocker", covering the calorimeter acceptance.

\subsection{Secondary background suppression}

Secondary sources of background events should also be considered. Such events are produced when secondary particles (photons, positrons, electrons) hit the materials inside and outside the target area. Special efforts should be devoted to install shielding wherever it is applicable. Also, since most secondary showers, born outside the target area, contain electrons and positrons, an additional suppression of such background can be achieved by installation of charge veto counters.

In the proposed configuration we investigate an installation of a tungsten rod (a "photon blocker") to dump a high flux of photons, emitted from the target at small angles outside the acceptance of the photon detector, and a thin scintillator, covering the colorimeter acceptance, to veto charged particles. Both elements are installed close to the exit of the dipole magnet - see Fig. 6 .

\subsection{Data Acquisition}

The expected rate of events with a threshold of minimum energy deposition in the calorimeter $E_{\text {cal }}>25 \mathrm{MeV}$ is about $450 \mathrm{kHz}$ for the projected luminosity of the experiment. Assuming a conservative value for the on-line suppression factor of 3 for combined veto-channels and for events with two or more photons in the calorimeter, one obtains a $150 \mathrm{kHz}$ final trigger rate.

It is clear that a conventional readout (based, e.g. on FASTBUS ADCs) does not meet the specifications. The front-end and digitizing electronics should be based on Flash ADC and FPGA logic to provide an on-line timing, cluster-finding and zero-suppression. Similar or substantially faster systems are now widely used or being designed for Data Acquisition of various experiments. However, at the moment the DAQ electronics for the described experiment is at an early stage of development.

\section{Monte Carlo}

In the proposed experiment we will search for a bump in a missing mass distribution on top of a QEDbackground. Some part of the background events can be identified and rejected; the remaining ones define the search sensitivity. For the study of the detector response to the background processes and for realistic 


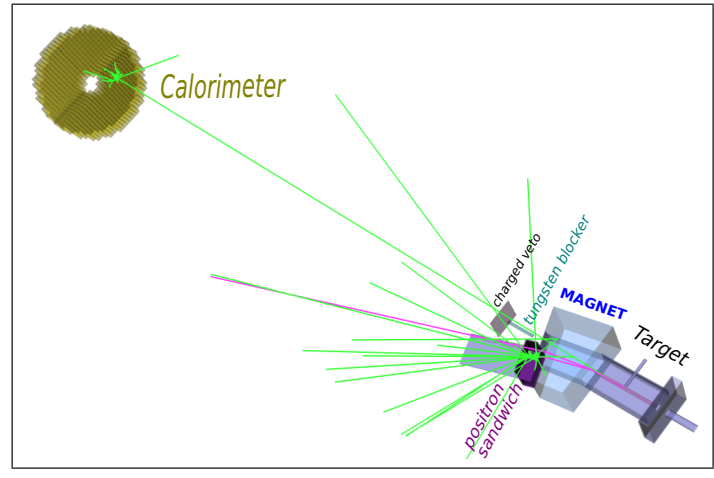

Figure 7. The final configuration accepted for the Monte Carlo simulation of the experiment.

estimation of the search sensitivity of the experiment, a detailed Monte Carlo simulation was performed using the GEANT4 toolkit. A set of dedicated event generators have been developed for the following processes:

1) positron bremsstrahlung $\quad e^{+}+\mathrm{H} \rightarrow e^{+}+X+\gamma$

2) radiative Bhabha scattering $e^{+}+e^{-} \rightarrow e^{+}+e^{-}+\gamma$

3) two-photon annihilation $\quad e^{+}+e^{-} \rightarrow \gamma+\gamma$

4) three-photon annihilation $\quad e^{+}+e^{-} \rightarrow \gamma+\gamma+\gamma$

5) signal process $\quad e^{+}+e^{-} \rightarrow \gamma+A^{\prime}$

The final configuration accepted for Monte Carlo is shown in Fig. 7.

To implement a bump search procedure one needs to know the experimental resolution for a reconstructed missing mass. This was determined from Monte Carlo. The missing mass resolution depends on the energy and angular resolution of the photon detector. Measured resolutions of the CLEO calorimeter, see ref. [5], were reproduced in our Monte Carlo by adding an equivalent of "electronics" noise to simulated energy depositions in the crystals. Noise parameters were taken from [5]. The simulation results for 4 values of $\mathrm{A}^{\prime}$-boson masses: 5, 10, 15 and 20 $\mathrm{MeV}$, when purely $\mathrm{A}^{\prime}$ production events were generated, are shown in Fig. 8. One can see that the missing mass resolution rapidly improves with increasing the masses of $\mathrm{A}^{\prime}$. The dependence can be fitted with an exponential function:

$$
\sigma_{\text {miss }}[\mathrm{MeV}]=3.6 \cdot \exp \left(-0.13 \cdot M_{A}[\mathrm{MeV}]\right) \text {, }
$$

which was used in the simulation of the search procedure. Note that such behavior means that a big tail from the two-photon annihilation $\left(M_{A}=0\right)$ is expected in a missing mass spectrum, and this indeed is observed in Monte Carlo. Therefore, for an efficient search at low $M_{A}$, a strong suppression of two-photon events is important.

Figure 9 shows the results of a full simulation for the same 4 possible masses of $\mathrm{A}^{\prime}$-boson. The kinetic-mixing parameter was set to $\epsilon=0.1$. The shown distributions are obtained for invisible decay of A' boson after all cuts are applied. For a designed experimental luminosity of $10^{33}$, each such spectrum can be collected in about 3 seconds.

\section{Projected search sensitivity}

The simulation data, presented in Fig. 9, allow us to carry out a realistic estimation of the search sensitivity. The search conditions are:

- Positron beam energy is $500 \mathrm{MeV}$.

- Luminosity is $10^{33} \mathrm{~cm}^{-2} \mathrm{~s}^{-1}$, which corresponds, for example, to a $30 \mathrm{~mA}$ beam current and a target thickness of $5 \times 10^{15}$ atoms $/ \mathrm{cm}^{2}$.

- Run time is taken to be $10^{7}$ seconds, which means a halfyear run with $65 \%$ time utilization.

- The search is performed using a sliding missing mass window with a width of $\pm 1.5 \sigma_{\text {miss }}$, where $\sigma_{\text {miss }}$ is evaluated using Eq.(1).

As expected, the search sensitivity is a function of missing mass range, and it exhibits a moderate dependence on the decay mode of the $\mathrm{A}^{\prime}$-boson and on the regime of operation of the veto counter. The best search sensitivity is demonstrated for invisible decay of $\mathrm{A}^{\prime}$ and veto-ON regime; the worst sensitivity is for a veto-OFF regime, but the difference is only a factor $\sim 2$.

Graphical representations of the projected search sensitivity are shown in Figures 10, 11.

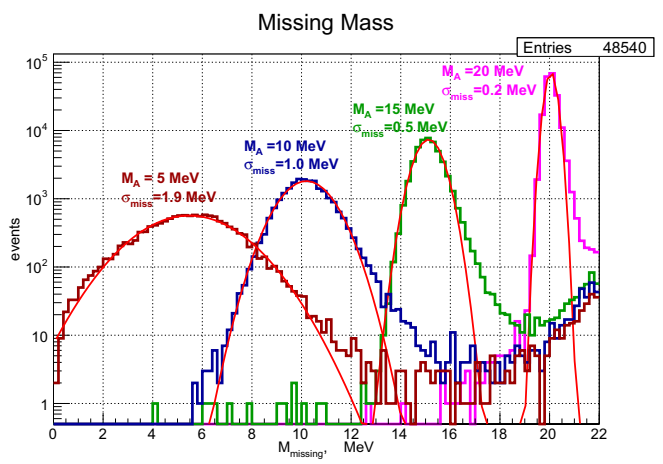

Figure 8. Missing mass reconstruction for 4 possible masses of $\mathrm{A}^{\prime}$-boson.
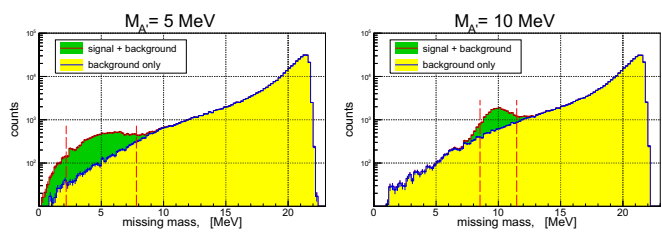

$\mathrm{M}_{\mathrm{A}^{\prime}}=15 \mathrm{MeV}$
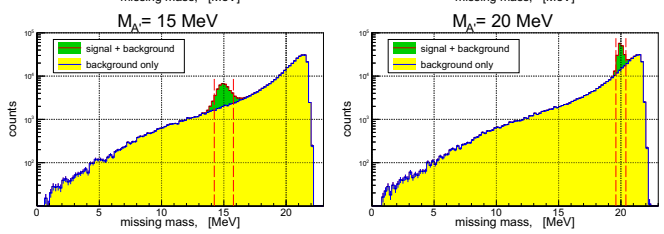

Figure 9. Full simulation of the experiment for 4 possible masses of $\mathrm{A}^{\prime}$-boson. Here the kinetic-mixing parameter $\epsilon$ was set to 0.1 . 
Table 1. Simulated search sensitivity.

\begin{tabular}{lc|cccc}
$\begin{array}{l}\mathrm{M}_{A^{\prime}} \\
\mathrm{MeV}\end{array}$ & $\begin{array}{c}\mathbf{M}_{\text {mis }} \text { window } \\
\mathrm{MeV}\end{array}$ & $\begin{array}{c}\text { veto } \mathbf{O N} \\
\text { invisible }\end{array}$ & $\begin{array}{c}\varepsilon^{2} \times \mathbf{1 0}^{\mathbf{8}}(95 \% \mathrm{CL}) \\
\text { veto } \mathbf{O N}\end{array}$ & $\begin{array}{c}\left(E_{e^{+}}+E_{\gamma}\right) \text {-cut } \\
\text { visible }\end{array}$ & $\begin{array}{c}\text { veto } \mathbf{\text { OFF }} \\
\text { visible }\end{array}$ \\
\hline 5 & \pm 2.8 & $\mathbf{9 . 7}$ & $\mathbf{1 5}$ & $\mathbf{1 3}$ & $\mathbf{1 7}$ \\
10 & \pm 1.5 & $\mathbf{8 . 7}$ & $\mathbf{1 5}$ & $\mathbf{1 2}$ & $\mathbf{1 5}$ \\
15 & \pm 0.8 & $\mathbf{5 . 2}$ & $\mathbf{1 1}$ & $\mathbf{8 . 2}$ & $\mathbf{9 . 1}$ \\
20 & \pm 0.3 & $\mathbf{2 . 4}$ & $\mathbf{5 . 9}$ & $\mathbf{3 . 3}$ & $\mathbf{3 . 5}$ \\
\hline
\end{tabular}

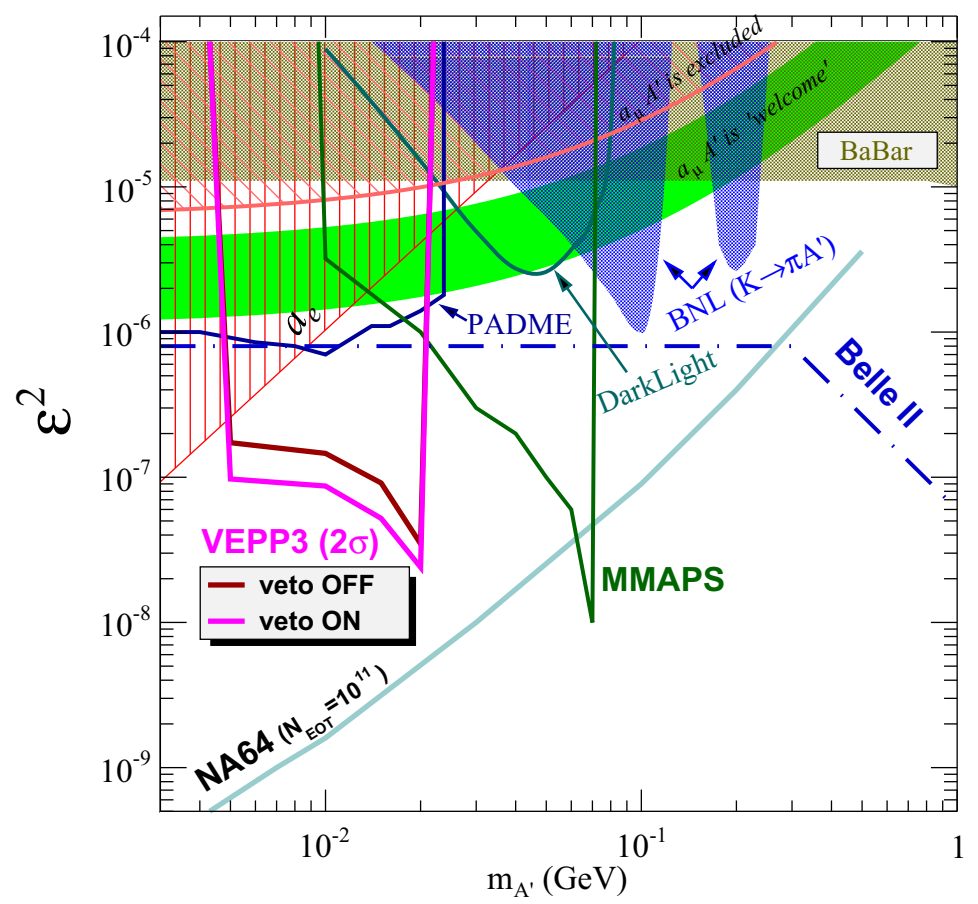

Figure 10. Projected parameter space and search sensitivity of the VEPP-3 experiment in the case of invisible decay of $A^{\prime}$. Shaded areas show existing bounds [8-10]. Curves demonstrate the search regions of the proposed experiments [6, 11-14].

\section{Conclusions}

- A decay mode independent search for a dark photon is effective in a setup with an intense circulating positron beam and an internal hydrogen gas target.

- At VEPP-3 such an experiment could be performed at the ByPass, which is now under development.

- Crystals from the CLEO endcap EM-calorimeter would be a good choice for the photon detector, but for optimal performance they should be equipped with fast photosensors and a sophisticated DAQ .

- Monte Carlo simulation shows that with such a setup in a half-year run, one can conduct a sensitive, decay mode-independent search in an interesting unexplored area of parameter space, where the "Atomki boson" could show up.

\section{References}

[1] B. Holdom Phys. Lett. B 178, 65 (1986).

[2] J. Alexander, et al., arXiv:1608.08632 (2016).

[3] B. Wojtsekhowski, arXiv:0906.5265 (2009).

[4] B. Wojtsekhowski, D. Nikolenko and I. Rachek, arXiv:1207.5089 (2012).

[5] Y. Kubota, et al., Nucl. Inst. and Meth. A 320, 66 (1992).

[6] J. Alexander (2016), URL https://indico.cern.ch/ event/507783/contributions/2150181/attachments/ 1266367/1874844/SLAC-MMAPS-alexander.pdf

[7] J. Alexander, private communication (2016).

[8] B. Aubert, et al., (BaBar Collaboration), arXiv:0808.0017 (2008).

[9] S. Adler, et al., (E787 Collaboration), Phys. Rev. D 70, 037102 (2004). 


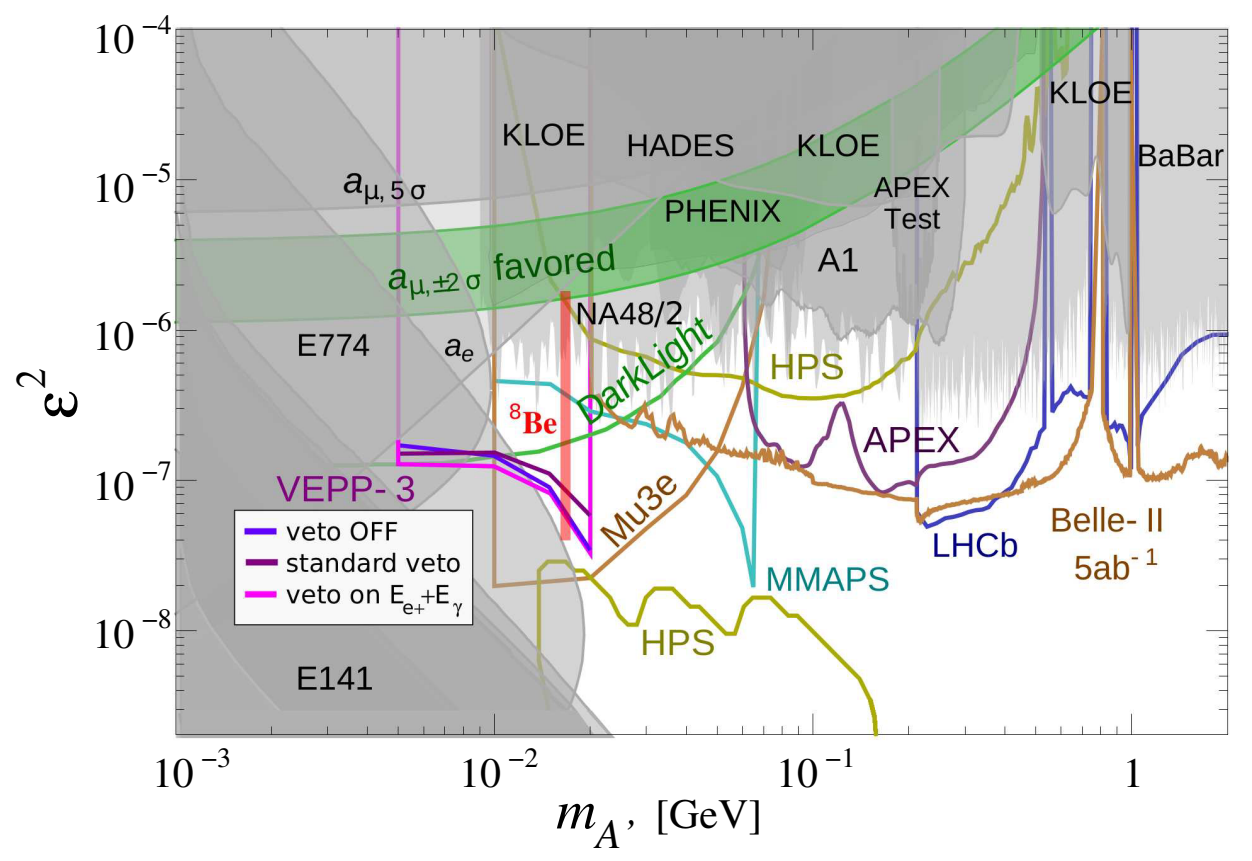

Figure 11. Projected parameter space and search sensitivity of the VEPP-3 experiment in the case of visible decay of $\mathrm{A}^{\prime}$ to $\left(e^{+} e^{-}\right)$-pair. Shaded regions show existing bounds; curves indicate the search areas of proposed experiments, see the review [2] and references therein. Note that the VEPP-3 search area covers almost the whole parameter space allowed for the putative "Atomki boson" [15], which is proposed to explain an anomaly in ${ }^{8} \mathrm{Be}^{*}$ decay [16].

[10] A. V. Artamonov, et al., (E949 Collaboration), Phys. Rev. D 79, 092004 (2009).

[11] M. Raggi and V. Kozhuharov, Adv. High Energy Phys. 2014, 959802 (2014).

[12] J. Balewski, et al., arXiv:1412.4717 (2014).

[13] R. Essig, J. Mardon, M. Papucci, T. Volansky, and Y.-M. Zhong, JHEP 11, 167 (2013).
[14] S. Andreas, et al., arXiv:1312.3309 (2013).

[15] A. Krasznahorkay, et al., Phys. Rev. Lett. 116, 042501 (2016).

[16] J.L. Feng, et al., Phys. Rev. Lett. 117, 071803 (2016), 